\title{
EFAD Statement on the Review Article - Research and Dietetic Response to COVID-19 in Europe: What Has Been Done and What Could Be Improved?
}

\author{
Eduard Baladia ${ }^{a}$ b Sharleen O'Reilly ${ }^{b, c}$ \\ ${ }^{a}$ Red de Nutrición Basada en la Evidencia, Academia Española de Nutrición y Dietética, Barcelona, Spain; ${ }^{b}$ Research and Evidence-based \\ Practice Committee, EFAD, Naarden, The Netherlands; ${ }^{C}$ UCD Institute of Food and Health, Dublin, Ireland
}

\section{Introduction}

In December 2019, the World Health Organization was alerted to a group of patients with pneumonia in Wuhan, and a month later the Chinese authorities confirmed that they had identified a new coronavirus, SARS-COVID-19. The pandemic began for most European countries in March-April, and since then our lives have dramatically changed. The public health response has been heavily dependent on the social, political, economic, and technological situation [1,2]; alongside the health system; emergency preparedness; and capacity [3].

The research response has been massive and unprecedented. From the calls for freely available, open access COVID-19 research [4] to the rapid launch of many studies aimed at understanding and treating the virus [5]. Undoubtedly, this research has resulted in important learnings on how to contain the pandemic, how the virus works and potential treatment routes [6]. However, this massive research response has generated a huge volume of peer reviewed and non-peer reviewed studies and commentaries, which potentially makes evidence-based practice and decision making more challenging, rather than easier.

\section{Research response to COVID-19: massive, poor quality and uncoordinated}

The volume of COVID-19 research generated in such a short period of time is unprecedented. Nowakowska [7] estimated that 2,062 articles were already published by March 31,2020. The majority $(55 \%)$ were published without peer review. They estimated that $36 \%$ of publications were commentaries, $34 \%$ were reviews (majority not systematic) and only $21 \%$ were original research [7]. Gianola [8] conducted another search two month later identify- ing 3,635 journal articles and 3,805 preprints. Surprisingly, only $9 \%$ of preprints were eventually published as scientific articles. They also found $<1 \%$ (both journals and preprints) were RCTs, and $<5 \%$ were systematic reviews [8]. Finally, Zyoud [5] found that by June 2020, 19,044 publications existed (in Scopus, which includes PubMed) and $48 \%$ were original articles. This points to a fivefold increase in publications in a single month and shines a clear light on the challenges presented by this exponential expansion. Prior to the pandemic, $85 \%$ of research could be considered as research waste due to a variety of issues such as poor research questions, poor study designs, inefficiency in the preparation, reporting and duplication of efforts [9]. The uncoordinated COVID-19 research has undoubtedly amplified these problems [10]. In fact, of the more than 2,000 clinical studies planned as COVID-19 treatments, only 2 were classified as adequate designs [11]. While no similar analysis exists for the field of nutrition, it is expected that the quality would be the same or potentially worse. Despite this necessary criticism, it is worth noting that the COVID-19 pandemic has also revealed the potential for modern global science to respond quickly, on multiple levels and across systems or fields of research [5]. Undoubtedly, as far as research is concerned, the need for prioritization and coordination systems has been highlighted to avoid duplication of efforts and waste in research [12].

\section{European Federation of the Associations of Dietitians (EFAD) and dietetic response across Europe: room for improvement}

The pandemic has shaken society to its core, but it has also highlighted the commitment of all health workers - including dietitians - to health systems and their communities. Dietitians are ex- 
perts in clinical nutrition who are highly qualified and legally regulated. They are the only professionals specifically trained to support the nutrition and hydration needs of acutely and chronically ill people and to monitor the nutritional status of populations at risk, including older adults and people with food insecurity [13].

Critically ill patients with COVID-19 admitted to intensive care units require nutrition, hydration and potentially medication delivered through feeding tubes, either enterally or parenterally [14]. Dietitians are experts in assessing individual patient nutritional demands considering their specific needs and circumstances. When people are well enough to leave hospital after having COVID-19, nutrition and hydration remain important. Dietitians have a role to play in rehabilitation, reducing risk of complications and shortening recovery, and they are key players in supporting delivery in practice. Outside of COVID-19 infection, the public health dietitian has a vital role due potential increased prevalence of food insecurity due to the wider impacts of COVID-19 on financial and social wellbeing [15]. It is also likely that eating behaviours may have worsened due to social isolation periods such as lockdowns [16] and these periods have made caring for people with pre-existence and nutrition-related diseases even more complex and difficult [17].

Professional bodies and institutions have responded in a time of unprecedented scientific uncertainty to try to support their members in providing evidence-based practice guidance. EFAD has taken a responsive approach to developing a variety of resources to support practice. The EFAD briefing document «The Role of Dietitians in Fighting COVID-19» outlined the central role that dietitians can and do play in the response to COVID-19. This role extends from the frontline in critical care to population-level advice. The paper also outlines the ability of the dietitian to provide support in acute community care, primary care, food service and education settings as well [13]. Dietitians have responded to the changes that COVID-19 has forced upon them and there is a need to capture the innovative ways with which dietitians have adapted their work practices and care delivery. EFAD would be well placed to coordinate this research across members and in turn, disseminate the findings widely so the implementation of best-practice can be modelled and adapted across Europe.

Through EFAD's responsive approach, it has positioned itself to be an information and support point for all European dietitians. EFAD (and member associations) have contributed COVID-19 resources to the EuDAP database in addition to actively disseminating resources through ESDNs, regular newsletter items and press releases. They also provided dietitians with access to eight free webinars for the continued training on COVID-19 related issues, such as the management of critical patients in ICU and re- habilitation, or how to deal with the pairing of cancer and COVID-19. Likewise, the EFAD website COVID-19 section is a trusted source of information for all European members. The website library is a collection of a wide variety of materials that have been gathered and sorted into cognate areas. In many cases, there are translated versions available tailored to the needs of each different European country and their dietitians. The resources include countries such as the United Kingdom, Ireland, Spain, Hungary, Turkey, Germany, Portugal, Greece, Israel, France, Netherlands, Turkey and Italy and they can all be accessed via the website (http://www.efad.org/en-us/covid-19/).

Despite the large efforts made by all European dietitians and the rapid response by EFAD and European Dietitian Associations, there remains a need for improvement. According to preliminary analysis by the EFAD Research and Evidence-based Practice Committee into the quality of resources made available to dietitians, the vast majority (83\%) of documents generated and shared had content intended to be «ready for use in practice» yet $98 \%$ were developed without following a systematic and transparent approach. It is also noteworthy that a considerable degree of duplication of efforts was detected. Consequently, the large amount of energy expended to produce guidelines, guides and resources has resulted a sizeable volume of work, however the quality of that evidence remains uncertain. This should give the dietetic profession food for thought. Should we be striving to develop better frameworks to support our responsiveness to situations like pandemics in the future? A strategic preparedness and response plan within EFAD could allow for greater coordination of future pandemic responses. It could also support higher standards for scientific evidence production being implemented so that systematic and transparent approaches prevail. This will ultimately lead to reduced research waste and improved translation of research into practice, which truly is the mission of dietitians everywhere.

\section{Summary}

- COVID-19 research has been exponential but of varying quality.

- EFAD has engaged a wide range of supports for dietitians to promote best-practice.

- Dietetic research into COVID-19 needs to be focused on improving the quality of the evidence base.

\section{Disclosure Statement}

The authors have no conflicts of interest to declare.

\section{References}

The references are available at www.karger.com/Article/Fulltext/513442. 